\title{
Wertschöpfungsketten neu denken und Barrieren überwinden
}

\author{
Seit 2014 entstehen immer mehr Läden, die Lebensmittel ohne \\ Einwegverpackungen - unverpackt - anbieten. Erste Forschungs- \\ ergebnisse zeigen, dass das Konzept wichtige Beiträge für einen \\ nachhaltigeren Konsum liefert, für dessen Erfolg aber Wertschöp- \\ fungsketten neu gedacht und gestaltet werden müssen. \\ Von Frederic Goldkorn, Melanie Kröger und Jens Pape
}

D as Aufkommen an Verpackungsabfällen steigt in Deutschland beständig und erreichte 2014 einen Spitzenwert von 17,8 Millionen Tonnen (Schüler 2016). Gleichzeitig ist das Land mit einer Recyclingquote von $71,4 \%$ Spitzenreiter im Umgang mit diesem Müll.

Jedoch räumt eine nachhaltige Wirtschaftsweise und auch die Abfallhierarchie des Kreislaufwirtschaftsgesetzes dem Vermeiden und dem Wiederverwerten von Abfällen Priorität vor dem Recycling ein. Die Zahlen zeigen aber, dass diese Vermeidung offenbar vernachlässigt wird.

\section{Entwicklung in Deutschland}

Ein Ansatz, dem Negativtrend der Verpackungszunahme unternehmerisch zu begegnen, ist die Eröffnung von Supermärkten, die Waren unverpackt bzw. in Mehrwegverpackungen anbieten. Inspiriert von ähnlichen Modellen in anderen Ländern sowie der sogenannten „Zero Waste“-Bewegung sind in Deutschland seit Februar 2014 mehr als 30 derartige Läden entstanden. Kunden nutzen hier ihre eigenen oder vor Ort erwerbbare Behälter, in die sie Waren füllen. Neben diesen „reinen“ unverpackt-Läden („Konzeptläden“) gibt es eine zunehmende Zahl an Läden, in denen ein Teil des Sortiments unverpackt angeboten wird („Streckenläden“).

Aufgrund des mittlerweile im Lebensmittelhandel etablierten hohen Grades an Verpackung und der damit verbundenen Abfallmengen birgt das „unver-
packt-Konzept“ ein großes Potenzial zur ökologischen Transformation der Prozesse. Gleichzeitig stößt es aber auf zahlreiche Herausforderungen. Im Rahmen des an der Hochschule für nachhaltige Entwicklung Eberswalde durchgeführten BÖLN-Projekts [1] „Der verpackungsfreie Supermarkt: Stand und Perspektiven. Über die Chancen und Grenzen des Precycling im Lebensmitteleinzelhandel“, wird gemeinsam mit den unverpackt-Läden daran gearbeitet, ebendiese Herausforderungen sichtbar zu machen, Handlungswege aufzuzeigen sowie die Vernetzung der Läden und die Ausweitung des Konzepts zu untersuchen und zu fördern.

\section{Herausforderungen}

Für den verpackungsfreien Kauf und Verkauf ist eine Umstellung von Handlungsmustern und Routinen entlang der Wertschöpfungskette notwendig. Hier gilt es, Lösungen zu finden, die auf allen Stufen anschlussfähig und praktikabel sind. Um solche Lösungen zu identifizieren, ist es nötig, das Konzept und die entsprechenden Herausforderungen aus verschiedenen Perspektiven zu betrachten.

Besonders in den Bereichen Vermarktung und Beschaffung zeigen sich spezifische Herausforderungen für das Konzept. In einer Zeit, in der Home Delivery, Convenience und to go-Produkte immer beliebter werden und der Lebensmitteleinkauf somit immer einfacher wird, gilt es, Kunden zu gewinnen, die bereit sind, ihr Einkaufsverhalten zu verändern und einen gewissen Mehraufwand zu betreiben. Außerdem gilt es, Produzenten von anderen Verpackungsmöglichkeiten $\mathrm{zu}$ überzeugen und Logistikketten umzustellen.

Ansätze des Sustainable-Supply-ChainManagement-haben in den letzten Jahren immer mehr Verbreitung in verschiedenen Bereichen der Logistik gefunden (Seuring/Müller 2013; Carter/ Easton 2011). Bezogen auf das Verpackungsaufkommen im Bereich der Lebensmittellogistik sind die Auswirkungen jedoch relativ gering. Im Fokus standen bisher die Optimierung der Transportmittel und der nahtlose Übergang von der Schiene auf die Straße. Außerdem werden die Reduzierung des Verpackungsaufkommens und die Vermeidung von Lebensmittelverschwendung häufig als konkurrierende Ziele betrachtet (Verghese et al. 2015).

Allerdings zeigen einige Naturkostgroßhändler erste Bemühungen zur Unterstützung des „unverpackt-Konzepts" in Form von Beratungsangeboten, höherer Flexibilität bei Gebindegrößen und der Bereitstellung von Verkaufsregalen und Behältern für unverpacktLäden.

\section{Verbreitung im bestehenden Lebensmittelhandel}

Die Gründer von unverpackt-Läden leisten wichtige Pionierarbeit in einem Feld, in dem Nachhaltigkeitsbemühungen entlang der Wertschöpfungskette bisher zumeist nur als Zusatznutzen ökonomischer Optimierungsprozesse auftraten (Corbett/Klassen 2006). Das Neudenken von Wertschöpfungsketten mit dem Ziel der Verpackungseinsparung bietet bei Überwinden der aufgezeigten Herausforderungen große Potenziale für ökologische Transformationsprozesse.

Auch die Aufnahme des Konzepts in bestehende (Bio-)Läden bietet Chancen, da diese über feste Kundenstrukturen verfügen und der zusätzliche Aufwand für die Kunden mutmaßlich geringer ist. Hier gibt es zum einen z. B. 
unabhängige Bioläden, welche ausgewählte Trockenprodukte wie Nudeln und Reis in Spendern zum selbst Abfüllen anbieten. Außerdem wurde das Konzept von der Biosupermarktkette „Bio Company“ übernommen, welche damit begonnen hat, neue Filialen mit einer „unverpackt“-Strecke auszustatten (Bio Company 2016).

Während eine solche Adaption des Konzepts durch etablierte Unternehmen und somit ein Übergang vom Nischenzum Massenmarkt zur Steigerung des ökologischen Impacts beitragen kann, stellt die dadurch zunehmende Konkurrenz für die aktuell innovationstreibenden Konzeptläden auch eine Herausforderung dar.

\section{Ladenbesitzer mit geringer Marktmacht}

Aufgrund ihres verhältnismäßig niedrigen Bestellvolumens sehen sich die einzelnen Ladenbesitzer in einer Position mit nur geringer Marktmacht. Um dies zu ändern und Prozessänderungen sowohl für Großhändler bzw. Logistiker als auch für Produzenten attraktiver zu machen, wird im Rahmen des Projekts ein Netzwerk initiiert.

\section{Anmerkung}

[1] Bundesprogramm Ökologischer Landbau und andere Formen nachhaltiger Landwirtschaft. Im Internet unter: www.bundesprogramm.de

\section{Literatur}

Bio Company (2016): Pressemitteilung vom 13.10.2016. Im Internet unter: http://www.biocompany.de/downloads/ pressemitteilung-bio-company-filiale-50.pdf

Carter, C. R./Easton, P. L. (2011): Sustainable supply chain management: evolution and future directions. In: International Journal of Physical Distribution \& Logistics Management 41/1:46-62.

Corbett, C. J./Klassen, R. D. (2006): Extending the horizons: environmental excellence as key to improving operations. In: Manufacturing \& Service Operations Management 8/1: $5-22$.

Kroll, C. (2015). Sustainable Development Goals: Are the rich countries ready? Gütersloh, Bertelsmann Foundation.

Schüler, K. (2016): Aufkommen und Verwertung von Verpackungsabfällen in Deutschland im
Jahr 2014. Studie im Auftrag des Umweltbundesamtes, Dessau-Roßlau.

Seuring, S./Müller, M. (2013): Nachhaltiges Management von Wertschöpfungsketten. In: Baumast, A./Pape, J. (Hrsg.): Betriebliches Nachhaltigkeitsmanagement. UTB. 245-258. Verghese, K. et al. (2015): Packaging's role in minimizing food loss and waste across the supply chain. In: Packaging Technology and Science 28/7: 603-620.

\section{AUTOREN + KONTAKT}

Frederic Goldkorn ist wiss. Mitarbeiter im Projekt unverpackt am Fachgebiet Nachhaltige Unternehmensführung in der Agrar- und Ernährungswirtschaft an der HNE Eberswalde, Schicklerstr. 5, 16225 Eberswalde. Tel.: +493334657337,

E-Mail: Frederic.Goldkorn@hnee.de, Website: http://hnee.de/unverpackt

Dr. Melanie Kröger ist wiss. Mitarbeiterin und Projektkoordinatorin im Projekt unverpackt am Fachgebiet Nachhaltige Unternehmensführung in der Agrar- und Ernährungswirtschaft an der HNE Eberswalde.

E-Mail: Melanie.Kroeger@hnee.de

Dr. Jens Pape ist Professor für Nachhaltige Unternehmensführung in der Agrar- und Ernährungswirtschaft und leitet das Projekt unverpackt an der HNE Eberswalde. E-Mail: Jens.Pape@hnee.de

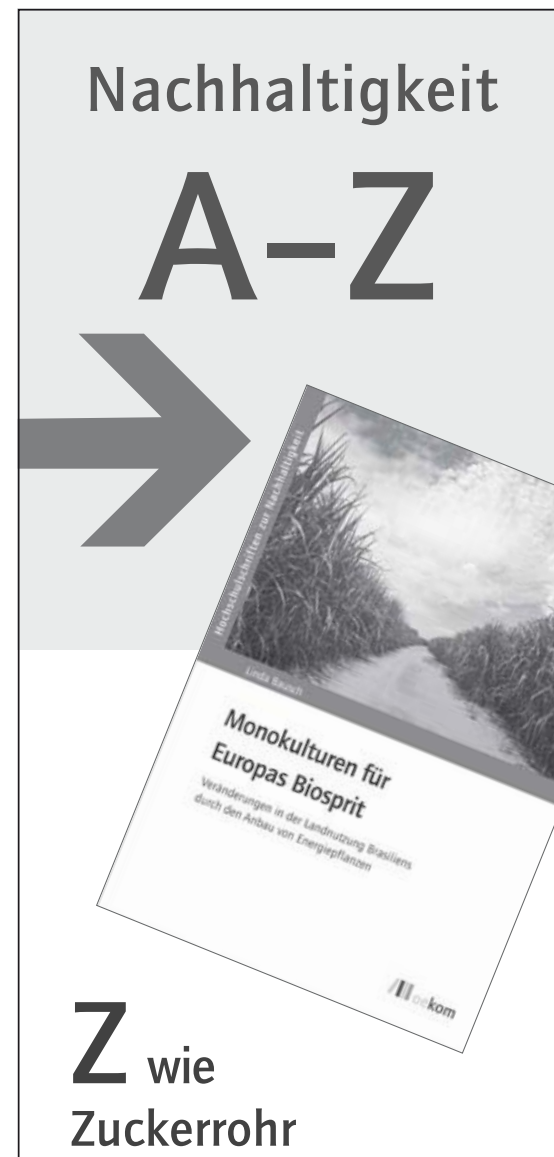

Kritiker haben es früh vorausgesehen: Eine EUBeimischungsquote für Biosprit führt auf der anderen Seite des Globus zu Monokulturen. Um europäische Autos ein bisschen klimaneutraler anzutreiben, werden die Probleme nach Brasilien verlagert und multinationalen Konzernen überlassen: Sie stehen dort für ungerechte Landverteilung, Rodung und einen nicht nachhaltigen Anbau von Zuckerrohr.

L. Bausch

Monokulturen für Europas Biosprit

Veränderungen in der Landnutzung Brasiliens durch den Anbau von Energiepflanzen Hochschulschriften zur Nachhaltigkeit Band 37 282 Seiten, broschiert, 29,95 Euro, ISBN 978-3-86581-816-4

Erhältlich im Buchhandel oder versandkostenfrei innerhalb Deutschlands bestellbar unter www oekom de. Auch als E-Book erhältlich.

\section{/II oekom}

Die guten Seiten der Zukunft 